\title{
Organic codes: A unifying concept to Life?
}

\section{Savio Torres de Farias ${ }^{1}$, Francisco Prosdocimi ${ }^{2}$ and Gustavo Caponi ${ }^{3}$}

${ }^{1}$ Laboratório de Genética Evolutiva Paulo Leminski, Centro de Ciências Exatas e da Natureza, Universidade Federal da Paraíba, João Pessoa, Paraíba, Brazil. https://orcid.org/0000-0002-2997-3629

${ }^{2}$ Laboratório de Biologia Teórica e de Sistemas, Instituto de Bioquímica Médica Leopoldo de Meis, Universidade Federal do Rio de Janeiro, Rio de Janeiro, Brazil. https://orcid.org/0000-0002-6761-3069

3 Departamento de Filosofia, Universidade Federal de Santa Catarina, Florianopolis, Brazil. https://orcid.org/0000-0002-3975-8367

\begin{abstract}
Although increasing knowledge about biological systems has advanced exponentially in recent decades, it is surprising to realize that the very definition of Life keeps presenting theoretical challenges. Even if several lines of reasoning seek to identify the essence of life phenomenon, most of these thoughts contain fundamental problem in their basic conceptual structure. Most concepts fail to identify necessary and sufficient features to define life. Here, we analyzed the main conceptual framework regarding theoretical aspects supporting life concepts, such as (i) the physical, (ii) the cellular and (iii) the molecular approaches. Based on ontological analysis, we propose that Life should not be positioned under the ontological category of Matter. Yet, life should be better understood under the top-level ontology of "Process". Exercising an epistemological approach, we propose that the essential characteristic pervading each and every living being is the presence of organic codes. Therefore, we explore theories in biosemiotics in order to propose a clear concept of life as a macrocode composed by multiple inter-related coding layers. Therefore, we suggest a clear distinction between the concept of life and living beings, a distinction that is not evident in theoretical terms. From the proposed concept, we suggest that the evolutionary process is a fundamental characteristic for life's maintenance but not to its definition. The current proposition opens a fertile field of debate in astrobiology, biosemiotics and robotics.
\end{abstract}

Keyword: Process, ontological category, life concept, essential feature

Corresponding author: Sávio Torres de Farias (stfarias@yahoo.com.br), Telephone number (+55 48 998370309). 


\section{Introduction}

The complexity and diversity of the life phenomenon has been fascinating humanity since the earliest records of human activity. Although classic explanatory models have been proposed by different human cultures to understand the origins and maintenance, it was along the year of 1859 that most these classic ideas were overcame. After the work of Charles Darwin, the view about how natural populations live, reproduce and migrate along the biosphere have gone through the most important paradigm shift in the history of natural sciences (Darwin, 1859). Besides other innovative ideas proposed by Darwin, the common descent among all life forms united all living beings into a singularity point in the past on which a very first form arose (Mayr, 2004). Since then, biology has experienced tremendous revolutions about mechanisms that characterize life. Despite our contemporary in-depth knowledge of intracellular biological mechanisms, it is amazing to realize that scientists have not achieved a clear and consensual definition of life yet. In our opinion this a conceptual and philosophical problem once researchers fail to pinpoint the two most important issues on concept formation: necessity and sufficiency. These basis for concept formation must be coped with the defining characteristics of life that must be both (i) shared among all living beings, and (ii) unique to them.

\section{Life as currently defined by physical, cellular and molecular approaches}

The conceptual perception about the life phenomenon faces an important duality: by one side, living and non-living entities can be clearly identified in our everyday life; on the other, we rather observe an enormous difficulty in the building of a strong concept 
that will make possible to identify the deepest essence of living entities (Bedau 1998; Cleland and Chyba, 2002; Dieguez, 2008; Dieguez, 2013). At least a dozen concepts describing what is life are currently under dispute, all of them rationally defensible as different researchers keep seeking to identify which characteristics are actually shared by all living organisms. Most of these concepts can be classified under three major conceptual approaches: (i) the physical; (ii) the cellular, and; (iii) the molecular.

Classical exponents of the physical approach are the Austrian physicists Ludwig Boltzmann (1844-1906) and Erwin Schrödinger (1887-1961); as well as the Russian physical chemist Ilya Prigogine (1917-2003). These researchers presented (along the years of 1877, 1944 and 1980, respectively) historically and intellectually relevant definitions of the life phenomenon evidencing its physical properties and considering life as a sort of physical sub-system. Among the features suggested, the physical approach focused essential and defining conditions for life in features such as: (a) the decrease in entropy, and (b) the distance from thermodynamic equilibrium. Even if life actually presents those characteristics implied by the defenders of the physical approach, these characteristics are not unique to biological systems. For instance, hurricanes and tornados can also be defined by presenting low entropy and distancing from thermodynamic equilibrium. Therefore, if one takes the physical approach as lifedefining, one must admit that life is merely a physical sub-system and, therefore, biological life is only one among different manifestations of such phenomena. Even if one takes this physical assumption for granted, a clear definition of what should be considered the biological or organic life keeps missing. Therefore, in order to find a concept of life restricted to the organic spectrum, we conclude that the physical approach does not meet the appropriate conditions to define life. More specifically, the physical approach is not a natural definition, but a compilation of characteristics shared by multiple 
physical systems that possess different origins and essences. The physical approach can therefore provide necessary conditions to define life, but not sufficient ones.

The second large life-defining approach is based on the premise that cells constitute the basic units of life. Over that premise, life becomes defined by characteristics presented by cellular entities. For instance, some of the characteristics often proposed by their advocates are: (i) self-replication capacity and autonomy; (ii) the presence of an informational program that drives both heredity and the metabolism (consisting in the interactions of cells with the environment); and, finally, (iii) the capability of evolving (Dieguez, 2008). Under a modern classic on this approach, the Spanish researchers Kepa Ruiz-Mirazo and collaborators (2004) proposed a universal definition of life based in both autonomy and open evolutionary capacity. A system that present these characteristics needs to have a semipermeable membrane, an energetic apparatus and two interdependent macromolecular components responsible for catalysis and information. The definition proposed by these authors (Ruiz-Mirazo et al., 2004) therefore evokes the same characteristics described by other approaches that take cells as the most basic units of life. By accepting these features as life defining, one assumes that organisms without cellular structures should not be classified as living. These ideas have taken viruses out of the tree of life, despite to the fact that viruses are capable to evolve, interact closely with living organisms and intertwin with the whole tree of life, playing an important role in biology (Farias et al., 2019). Recent works in the virosphere are clarifying the enormous relevance of virus to Earth's biosphere (Zhang et al. 2018; O’Malley, 2016; Lang et al., 2009; Suttle 2007), to the evolution of cellular organisms (Dupré and Guttinger, 2016; Pradeu, 2016), to the development of organs and tissues (Chuong, 2018), to the packing of chromatin (Sosa et al., 2013), etc. 
Even if the removal of viruses from the list of living organisms could not be seen as a strict conceptual problem, other borderline examples do actually challenge the cellular-based definition of life. For instance, non-fertile hybrids such as the mule do not have the capacity for self-replicating and, therefore, they do not form lineages, being outside the evolutionary process. Nevertheless, the mules are clearly alive. It follows that, if one accepts that it is not necessary to identify all cell-based characteristics to consider an entity as living, but barely a subset of them, why should viruses be considered not living entities? At least, they can form lineages and evolve... This conceptual impasse clearly demonstrates that exist theoretical problems in constructing a unifying concept of life that take cells as the basic units of concern.

The third, molecular-based approach has flourished in the last decades due to the increased knowledge about the molecular functioning of living beings and due to $\mathrm{XXI}^{\mathrm{st}}$ century's assumptions about the origin of life. This approach gained argumentative force since the 1980s with the discovery of catalytic activity in RNA molecules (Kruger et al., 1982, Guerrier-Takada et al., 1983). RNA has been proven capable to both (a) store hereditary information, and (ii) perform catalysis (metabolism), two essential characteristics for life. The centrality of RNA molecules in the process of life formation reached its high point with the proposition of an RNA-World. Under this widespread scenario, RNA molecules initially organized the essential processes that determined the differentiation between the living and the non-living (Gilbert, 1986, Poole et al., 1998, Jeffares et al., 1998, Dworkin et al., 2003, Lazcano, 2012, Reyes-Prieto et al., 2012, Neveu et al., 2013). In line with this proposal, Gerard Joyce (1994) elegantly proposed to conceptualize life as a self-sustaining chemical system that is capable of Darwinian evolution. This definition did not assume the existence of cellular organization, as Joyce (1994) stated that these characteristics were already present in the world organized by 
RNAs. He therefore suggested that the essence of life was self-replication with modification. It must be noticed however that the notion that life should be understood as "self-replication with modification" is quite similar to the cellular argument; with a temporal regression to the molecular line that gave rise to the cells. Thus, we must keep in mind that similar criticisms regarding the life of infertile hybrids should be applied here. Infertile mules, for instance, are alive in spite of being unable to participate in the evolutionary process by "self-replicating" themselves. Additionally, the process of reproduction (replication) in the RNA world could be reduced to an ordinary process of chemical reactions between atoms and molecules, ceasing to present any particular feature of a biological system.

Almost getting to the point, two recent works (Lanier et al., 2017; Vitas and Dobovisek, 2018) propose that the main essence of life is a collaborative connection between the two main constituent molecules of the process, $i$. e., peptides and nucleic acids. They missed however the identification about precise point on which chemistry become biology and did not aim to provide any precise definition of life.

In 2002, a stimulating publication by Cleland and Chyba (2002) faced the conceptual limitations that life definitions presented and questioned whether life should be a natural kind. In philosophy, natural kinds are considered to be material entities capable to be defined by unique, distinctive characteristics. These authors suggested that either the development of an artificial living system in the laboratory or the discovery of life on another planet would be important counterpoints to the establishment and elaboration of a unifying concept. Contrarily, Dieguez (2013) held that life could be indeed characterized as a natural kind; however, the researcher pointed out that the central problem in this conceptualization is the search for an essentialist definition. Dieguez 
therefore proposed a non-essentialist view defining life as a group that contained homeostatic properties. Yet, this author did not make clear which properties should be considered and how one should identify them.

In the last paragraphs, we have evidenced conceptual difficulties in the most accepted scenarios currently used to define life. So, where should we go now? Where should it be the emergent principle that unequivocally characterizes life? Can we find it? How should we define it?

\section{Chasing strict conceptual constructions to define life}

The use of a rational process to construct strong concepts requires that certain specific criteria should be obeyed in order that the invariant characteristics of the object (or process) under definition can be clearly evidenced, allowing its unambiguous discrimination from other existing things.

In this sense, any proposed model lose support in the case on which the delimitations provided were mistaken, incomplete or erroneous. We may think about a "starting-point error" when misleading a priori definitions guides us to the search for characteristics intended to justify a given preconceived conclusion; avoiding us to understand the deep essence of the entity or process that needs to be conceptualized. With this in mind, a close reading of the aforementioned approaches used to define life lead us to appreciate that both cell and molecular approaches are contaminated with this startingpoint error. These indications may, to some extent, allow us to understand why these approaches failed to provide a precise identification of life's essence: this occurred 
because the characteristics defined a priori limited the exploratory power of the phenomenon.

The fact that previous proposals to identify a unifying concept for life have failed does not mean that this quest cannot be clearly elucidated. Thus, our approach here aims to analyze the general processes observed in living beings and to inquiry for characteristics shared by all of them in such a way that theses process and characteristics can reflect their deep essence and the principle of the life phenomenon.

\section{Top-level ontological classes for defining the life phenomenon}

Before going deeper in the quest for a new proposal to conceptualize life, it is necessary to analyze the theoretical organization that has been used as basis for its conceptual construction. The three large conceptual schemes based on (i) physical, (ii) cellular, and (iii) molecular characteristics provided a theoretical distinction between living and non-living that deals with material entities. But what if life has been mistakenly classified in the ontology of Matter? Which other higher-level ontologies do we have available to characterize the life phenomenon?

Although many different ontological trees exist, an interesting work by Chi and collaborators (1994) depicted an ontological tree with three main categories: (i) Matter, (ii) Processes, and (iii) Mental States (Figure 1). At this point, it is interesting to notice an apparent paradox between (a) the act of separating and identifying the living from the non-living, and (a') what we can properly understand and conceptualize as life. At (a), the observation of the constituent parts of the entities will inform us about the living (or non-living) nature of these entities. This happens because living beings clearly belong to 
the category of Matter and they can be easily identifiable and distinguished. However, the search for identifying the essence of life based on material characteristics has never been trivial and presents an actual challenge to the construction of this concept.
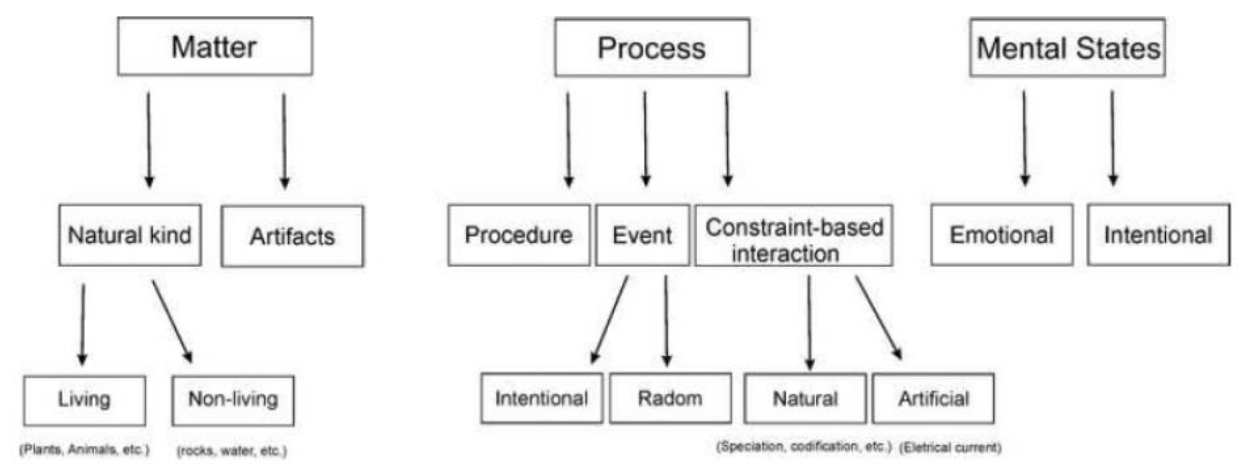

Figure 1 The ontological categories in which all the entities of the world may belong. Modified from Chi et al 1994.

The question pointed by Cleland and Chyba (2002) on whether life would be a natural kind is part of the presupposition that the life phenomenon is inserted within the ontological class of Matter. We must consider that the correct framing of an entity within a top-level ontological category is a primordial step towards the construction of a clear 
definition, given to the fact that different top-level ontological categories devise particularly different principles and essences.

As mentioned earlier, we can either admit life as a natural kind or not. Researchers have always admitted that, but what if we decide to challenge this view? This intellectual confront will lead us into two alternative interpretations: by one side, (i) we may suppose that the essence of life is found in the ontological category of Matter, but were unable to show it clearly until this moment, or; (ii) maybe the essence of life cannot be found in the ontological category of Matter, and, therefore, it cannot be evidenced as a natural kind. In two important works, the cognitive scientist Michelene Chi and her collaborators (Chi et al.,1992; Chi and Roscue, 2002) suggested that, during the learning processes, misunderstandings about the nature of things often happen due to a misinterpretation about their top-level ontological category, leading to erroneous interpretations. This is exactly the problem we identified in the conceptualization of the life phenomenon. Therefore, the difficulties faced by philosophers are not related to the lack of an identifiable essence, but to a mistaken ontological positioning as we will argue that life may not belong to the top-level ontological hierarchy of Matter.

We therefore propose that life should be understood under the ontology of Processes. Even if living beings are clearly material entities, they exist due to the continuity of a process. Also, as a consequence, the interruption of this process is directly related to the cessation of the living matter. Thus, we understand that living beings consist in the materialization of a Process and this Process is the actual essence of the life phenomenon.

Under this new interpretation, life should now be characterized under some subdivision of the Process category. Although it might be also understood as either an 
"Event" or a "Procedure", probably the better description should be "Interaction based on constraints" (Figure 1). By constraints we understand rules or conventions provided by the grammar of the universe, as the rules of Physics and Chemistry. Now, we must identify which biological processes meet these required conditions and check whether they can really support the ontological class replacement. Consequently, in the case these processes demonstrate to be universal, unique and exclusive to living beings; we will use them as the best descriptors of the essence we have been looking for. This way, we aim to achieve a higher theoretical precision for defining the phenomenon of life.

\section{Organic Codes: The Processual Essence of Life}

Traditionally, biology focuses its studies in the understanding of biological things or entities, such as the study of cells, individuals and species. Under this historical method of analysis, things precede the processes that maintain them (Dupré and Nicholson 2018). However, some authors have been proposing a modification in that interpretation, moving from the sphere of things to focus on processes, once the living world presents an identifiable hierarchy of such processes (Dupré 2012, Dupré and Nicholson 2018, Simons 2018). Dupré and Nicholson (2018) argue that even entities can be understood as specific temporal stages of stable processes. It is the spatiotemporal organization processes, together with their causal and temporal relationship with other processes that will determine their stability and persistence. In line with these thoughts, Simons (2018) suggested that material entities should be understood as some sort of precipitating of the processes that maintain and stabilize them. Following a similar line of reasoning, we propose that living beings should be understood as the materialization of the process that is essential for their maintenance and stability. The repositioning of the life phenomenon 
from the category of Matter to the category of Processes is essential to achieve this acknowledgement. A direct consequence of this replacement is the modification on the way through which living beings will start to be analyzed and understood. This way, it ceases to be necessary to look for material causes or search common features shared by living beings. Under this new approach, it becomes necessary to look for general laws that determine their deepest functional logic. Then we come into the two main characteristics classically identified as Processes often highlighted as essential for life, $i$. e., (i) metabolism, and (ii) heredity.

By (i) metabolism, we understand the set of chemical reactions (catalyzed either by proteins or RNAs) that recycle the basic monomers of biopolymers and provide energy to the synthesis of essential compounds necessary to the maintenance of life. The principle of metabolism is the transformation of compounds throughout the processes of degradation and synthesis. While these processes of transformation are of extreme relevance for the maintenance of living beings, they are not unique to them as many chemical and physical processes are likewise based on transformations. On the other hand, (ii) heredity may be understood as the process of genetic material transference between generations along lineages of living organisms. We therefore must comprehend that the main pillar of heredity is the process of replication of the genetic material replication. This process can be reduced into a set of chemical reactions catalyzed by proteins and their cofactors, being also a metabolic process.

If we consider life under its new ontological status, we start to glimpse overlapping layers of organization shared by all living beings that are responsible to maintain their Processes functioning. The understanding that living beings are composed by multiple layers of codes has been a fertile ground for the research field of Biosemiotics 
(Barbieri 2012; Barbieri, 2013; Barbieri, 2016; Barbieri, 2017; Hofmeyr, 2018). Biosemiotics researchers are focused in understanding how communication happens in biological organisms through their encoded systems. Their studies have established important characteristics identified on biological codes, such as: (i) the code must connect two words, one defined as the signal and other described as the biological sense; (ii) a code must have an adapter that establishes the rules of correlation and makes contact between the signal and its meaning; and, (iii) the code must be a natural convention; even if the information is changed experimentally, it must still act as a code (Hofmeyr, 2018).

Several structures in biological systems work in codified forms. At the molecular level we could describe, for instance: the genetic code, the regulation of gene expression, the hormone-receptor signaling system, the histone code, the epigenetic code. And, at higher levels, other codes can be described, such as: language, sexual selection, social conduct; amongst many others. It is important to notice that coding systems exist only in biological systems or in systems directly derived from biological organisms, such as in the case of language or computer programs. When analyzing the origin and evolution of codes, it is possible to glimpse that the overlap of new codes over earlier/ancient ones often increase the complexity of the previous codes, operating to better adjust and fine tune them. These inter-related codes start to interact in order to form a macrocode composed of the multiple, overlapping coding systems (Barbieri, 2014). The presence and integrated relationship between these multiple coding layers is the fundamental feature that defines the organization of living beings. 


\section{Open and closed codes}

Before going further, it seems important to explicit the concept of information we are assuming here. Many previous works have described difficulties to precisely define what is information (Hoffmeyer and Emmeche, 1991; Capurro and Hjørland, 2003). Therefore, in order to proceed with our explanation, we aim to consider information as "the establishment of a connection between two or more natural entities". This connection will become "encoded information" when it presents an intermediate entity responsible for coding and/or decoding.

Among coding systems, it is possible to recognize two essential types of codes: the (i) open codes, and the (ii) closed codes. In the open codes, the processed information can be adjusted to "user's" needs; while in the closed codes, the information cannot be adjusted. We understand that the phenomenon of life can be materialized as either open or closed codes. The organic codes should be considered as open when organisms are target of the evolutionary processes. In that case, the evolutionary process acts as the "user" that presents new conditions and adjusts the information necessary to the maintenance and perpetuation of the coding system. Nevertheless, living beings can also be materialized as closed coding systems when the evolutionary process in uncapable to adjust the system. For instance, infertile hybrids are living systems with closed codes as they hold the organic codes necessary to process organic information; even if they cannot constitute lineages and be targets of the evolutionary process. 


\section{A code-dependent information system}

From the assumptions presented, we propose a primary definition of life as a codedependent information system. In 1998, the Danish theoretical biologist Claus Emmeche proposed that the concept of life should be based in the code-structural nature of biological systems. For this author, "life is the functional interpretation of signs in selforganizing material 'code-systems' that construct their own 'umwelts'"' (Emmeche, 1998). We were inspired by his works and follow a similar line of reasoning. On the other hand, our proposal is distinguished from Emmeche's because his ideas presuppose a materialization of the process. Instead, our proposal is more metaphysical and place the definition of life into the ontological category of Process.

When considering that biological systems consist on overlapped macrocodes, we may wonder if each coding layer should be understood as individualized living systems? The answer is no because the overlapping of codes creates an inter-related functional system of correspondence that gives rise to the emergence of a macrocode. In order to better define in which case a code alone can be considered alive, we present a derivation of the primary concept. In this case, life should be understood as an information system that is dependent of a code (open or closed) for its processing but operates independently of other macrocode structures. To better illustrate this issue, let us consider the language, which is clearly an information system processed by a code. As we defined, language alone cannot be considered alive once its functioning is dependent of other macrocode structure, i. e., the cognitive code in the human brain. Although language does evolve under Darwinian-like ways, it is strictly dependent on other codes and should be considered as a living system. 
In a biological context, the emergence of a first encoded processing system at the molecular level sparkled the phenomenon of life. The further appearance of new processing systems increased the complexity of the whole structure and allowed the development of emerging properties every time a new coding layer was incorporated into the biological systems.

\section{The role of evolution in the definition of life}

The initial understanding about the role of the evolutionary process in the history of living beings came from the work and ideas of Charles Darwin. His ideas drastically modified the traditional way on which most people understood the natural world and the essence of living beings. Since then, it is impossible to understand biology if we detach ourselves from evolutionary explanations. The explanatory power of the Darwinian thought and its further developments has been confirmed with enormous robustness along different fields of specialization as they found the fundamental axis for conceptual construction in biology.

This robust explanatory power often leads to a synonymy between the origin of life and the origin of the evolutionary process (Joyce, 1994). In many investigative lines, the evolutionary process is considered the defining characteristic of life (Joyce, 1994, Reyes-Prieto et al., 2012). Due to the pivotal relevance of Darwinian-based evolution, we must analyze its role in the conceptualization of life proposed here.

In both cellular and molecular approaches, characteristics such as autonomy and self-sufficiency have been invoked. These features assume an evolutionary process, including evolution as a defining characteristic of life. Even though, we propose here to 
define life as an information system dependent on codes for its processing. In this context, we can identify two types of codes: opened and closed, being the open subject to adjustments by the evolutionary process. One of the essential conditions for demonstrating the relevance of the evolutionary process is the establishment of lineages. In the present context, however, when defining life as a macrocode, we were astonished to conclude that the evolutionary process leaves to be an essential characteristic of life. But this astonishment is surpassed when we figure out the fact that closed organic codes must be considered alive; such as in the cases of infertile organisms incapable to establish lineages. Nevertheless, the evolutionary processes keep being a necessary characteristic for the perpetuation and maintenance of open codes, being an intrinsic characteristic of this type of coding systems. In this sense, the evolutionary process should be better understood as a need for the maintenance of continuously changing forms of life under dynamic environments than a strict requirement for the existence of life.

\section{Last considerations}

After we scrutinized the conceptual structures historically used to define life and reorganized the concept of life under a new top-level ontology, inevitable outcomes should be considered. First, we must acknowledge the separation between (a) life and (a') living beings. Under different conceptual perspectives, we observe an overlap between these two classes; a tendency that is more evident under the cellular approach - on which life is the cell and the cell is the living being "par excellance". Our approach tries to circumvent this problem by proposing a metaphysical concept for life. For us, life should be understood as an independent process defined by multiple coding layers. Thus, living beings should be understood as the materialization of this encoded process that 
characterizes life. The most important macrocodes on which life is based are open codes that are subject of modifications along time and other (ecological) conditions imposed.

The conceptual adjustment proposed here opens new avenues for theoretical and practical exploration, with implications in areas such as astrobiology and computer science. In astrobiology, it provides new insights to the quest for life outside Earth once it establishes a non-typological parameter for life without resorting on how the process may be materialized under different cosmic conditions. In the computer sciences and robotics, it provides a guideline for the establishment of a decision criteria to define what should be considered an artificial life.

The view of life as an information system dependent of a code that operates independently of other macrocode structures, allows a processual definition of life comprising necessary and sufficient features to define the life phenomenon. On one side, the use of this definition allows that both infertile organisms and virus come to be considered as living beings. On the other, it excludes human-created codes such as language or computer-generated codes from being considered alive. The current proposition avoids the misuse of the term Life, clarifies historical misunderstandings about the concept and provides a bona fide logical structure to basis this extremely relevant concept.

\section{Acknowledgment}

STF was supported for Coordenação de Aperfeiçoamento de Pessoal de Nível Superior (CAPES) and the Program of Pos-graduation in Philosophy at the Universidade Federal de Santa Catarina, Brazil. FP was supported by Fundação de Amparo à Pesquisa 
do Estado do Rio de Janeiro (FAPERJ, CNE E-26/202.780/2018) and by the Conselho Nacional de Desenvolvimento Científico e Tecnológico (CNPq, PDE 205072/2018-6).

\section{References}

- Barbieri, M., (2012). Code biology - A new science of life. Biosemiotics. 5: 411- 437.

- Barbieri, M., (2013). The paradigms of biology. Biosemiotics. 6: 33 -59.

- Barbieri, M., (2014). Introduction to code biology. Biosemiotics. 7: 167 -179.

- Barbieri, M., (2016). From the common ancestor to the first cells: the code theory. Biological Theory. 11: 102-112.

- Bedau, M.A., (1998). Four puzzles about life. Artificial life. 4: 125- 140.

- Boltzmann, L., (1877). Über die Beziehung zwischen dem zweiten Hauptsatze der mechanischen Wärmetheorie und der Wahrscheinlichkeitsrechnung respective den Sätzen über das Wärmegleichgewicht. Wien. Ber., 76 (1877), pp. 373-435.

- Capurro, R., Hjørland, B., (2003) The Concept of Information. In: Annual Review of Information Science and Technology. Ed. B. Cronin, Vol. 37 (2003) Chapter 8, pp. 343411.

- Chi, M.T.H., Roscoe, R.D., (2002). The processes and challenges of conceptual change. In: M. Limón \& L. Mason (Eds.), Reconsidering Conceptual Change. Issues in Theory and Practice, 3-27. 
- Chi, M.T.H., Slotta, J.D., Leeuw, N., (1994). From things to process: A A theory of conceptual change for learning science concepts. Learn instruction. 4:27- 43.

- Chuong, E.B., (2018). The placenta goes viral: Retroviruses control gene expression in pregnancy. PLoS Biology. 9;16(10):e3000028.

- Cleland, C.E., Chyba, C.F., (2002). Defining 'life'. Origin of Life and Evolution of Biospheres. 32(4):387-93.

- Dieguez, A., (2008). Es la vida un género natural? Dificultades para lograr una definición del concepto de vida. ArtefaCTos. 1: 81-100.

- Dieguez, A., (2013). Life as a Homeostatic Property Cluster. Biological Theory. 7: 180 $-186$.

- Dworkin, J.P., Lazcano, A., Miller, S.L., (20030. The roads to and from the RNA world. Journal of Theoretical Biology, 7, 222, 127-34.

- Dyson, F., (1985). Origins of Life (Cambridge U. Press).

- Dupré, J., (2012). The Constituents of Life 2: Organisms and Systems. In J. Dupré, Processes of Life Essays in the Philosophy of Biology. (pp. 85-100). New York, United States of America.

- Dupré, J., Nicholson, D., (2018). A manisfesto for a processual pilosophy of biology. In D. J. Nicholson \& J. Dupré (ed), Everything flows: Towards a processual philosophy of biology. (pp. 3-45), Oxford, United Kingdom.

- Dupré, J., Guttinger, S., (2016). Viruses as living processes. Studies in History and Philosophy of Biological and Biomedical Science, 59, 109-116 
- Emmeche, C., (1998). Defining life as a semiotic phenomenon. Cybernetics And Human Knowing, Vol.5, no.1, 1998, pp. 3-17.

- Farias, S.T., Jheeta, S., Prosdocimi, F. (2019). Viruses as a survival strategy in the armory of life. History and Phylosophy of Life Science. 41, 45 (2019). https://doi.org/10.1007/s40656-019-0287-5

-Frege, G., (1971). Ecrits logiques et philosophiques. Trad. e Introd.: C. Imbert. Paris: Editions du seuil.

- Gilbert, W., (1986). Origin of life: The RNA world. Nature. 319 (6055): 618-618.

- Guerrier-Takada, C., Gardiner, K., Marsh, T., Pace, N., Altman, S., (1983). The RNA moiety of ribonuclease $\mathrm{P}$ is the catalytic subunit of the enzyme. Cell. 35(3 Pt 2):849-57.

- Hofmeyr, J.S., (2018). The first Special Issue on code biology - A bird's-eye view. Biosystems. 164:11-15. doi: 10.1016/j.biosystems.2017.12.007.

- Hoffmeyer, J.S., Emmeche, C., (1991). Code-duality and the semiotics of nature. In: Myrdene Anderson and Floyd Merrell, eds. On Semiotic Modeling. Mouton de Gryter, Berlin \& New York pp. 117-166.

- Jeffares, D.C., Poole, A.M., Penny, D., (19980. Relics from the RNA world. Journal of Molecular Evolution, 46, 18-36.

- Joyce, G.F., (1994). The Rna World: Life Before Dna and Protein, in ExtraterrestrialsWhere Are they? II, ed. B. Zuckerman, M. Hart. Cambridge: Cambridge U. Press. 
- Kruger, K., Grabowski, P.J., Zaug, A.J., Sands, J., Gottschling, D.E., Cech, T.R., (1982). Self-splicing RNA: autoexcision and autocyclization of the ribosomal RNA intervening sequence of Tetrahymena. Cell. 31(1):147-57.

- Lang, A.S., Rise, M.L., Culley, A.I., Steward, G.F., (2009). RNA viruses in the sea. FEMS Microbiology Review, 33(2), 295-323.

- Lanier, K.A., Petrov, A.S., Williams, L.D., (2017). The Central Symbiosis of Molecular Biology: Molecules in Mutualism. Journal of Molecular Evolution. 85(1-2):8-13. doi:10.1007/s00239-017-9804-X.

- Lazcano, A., (2012). The biochemical roots of the RNA world: from zymonucleic acid to ribozymes. History and Philosophy of Life Science, 34, 407-23.

- Neveu, M., Kim, H.J., Benner, S.A., (2013). The "strong" RNA world hypothesis: fifty years old. Astrobiology, 13, 391-403. doi: 10.1089/ast.2012.0868.

- O’Malley, M.A., (2016). The ecological virus. Studies in History and Philosophy of Biological and Biomedical Science, 59, 71-79

- Poole, A.M., Jeffares, D.C., Penny, D., (1998). The path from the RNA world. Journal of Molecular Evolution, 46:1-17.

- Prigogine, I., (1980). From Being to Becoming. SanFrancisco: W. H. Freeman.

- Reyes-Prieto, F., Hernández-Morales, R., Jácome, R., Becerra, A., Lazcano, A., (2012).

Coenzymes, viruses and the RNA world. Biochimie, 1, 94, 1467-73. doi: 10.1016/j.biochi.2012.01.004. 
- Ruiz-Mirazo, K., Peretó, J., Moreno, A., (2004). A universal definition of life: autonomy and open-ended evolution. Origin of Life and Evolution of Biospheres. 34(3):323-46.

- Simons, P., (2018). Process and precipitates. In D. J. Nicholson \& J. Dupré (ed), Everything flows: Towards a processual philosophy of biology. (pp. 49-60), Oxford, United Kingdom.

- Sosa, D., Miramontes, P., Li, W., Mireles, V., Bobadilla, J.R., José, M.V., (2013). Periodic distribution of a putative nucleosome positioning motif in human, nonhuman primates, and archaea: mutual information analysis. International Journal of Genomics. 2013:963956.

- Suttle, C.A., (2007). Marine viruses-Major players in the global ecosystem. Nature Review in Microbiology, 5, 801-812.

- Schrödinger, E., (1944). What is life? The physical aspect of the living cell. Cambridge university press.

- Vitas, M., Dobovišek, A., (2018). In the Beginning was a Mutualism - On the Origin of Translation. Origin of Life and Evolution of Biospheres. 48(2):223-243. doi:10.1007/s11084-018-9557-6.

- Zhang, Y.Z., Shi, M., Holmes, E.C., (2018). Using metagenomics to characterize an expanding virosphere. Cell, 172(6), 1168-1172. 